\title{
Impacto económico de las consecuencias de la variación de las condiciones de la concesión de la AP-7 en la Comunidad Valenciana a partir del año 2020.
}

\author{
José Enrique Pérez Zamora \\ Ingeniero Civil. España \\ Miguel Ángel Carrera Hueso \\ Ingeniero de Caminos, Canales y Puertos.
}

Jefe de sección de proyectos de carreteras de la Consellería de Vivienda, Transporte y

Vertebración del Territorio de la Generalitat Valenciana.

Profesor asociado de la Unidad Docente Caminos y Aeropuertos de la Universidad

Politécnica de Valencia. España

\section{RESUMEN}

La autopista AP-7 forma parte del corredor viario del mediterráneo, uno de los grandes corredores de la Unión Europea, y comunica Francia con Algeciras. En la actualidad, gran parte de la misma dentro de la Comunidad Valenciana se encuentra en régimen de concesión, que se extingue a finales de 2019.

Esta situación futura creará un impacto económico en dicho territorio que merece ser estudiado desde el punto de vista técnico. Para ello, se realiza un estudio del tráfico actual y una prognosis hasta el año 2040, donde se plantean diferentes escenarios basados en las transferencias del tráfico hacia la AP-7 desde ejes paralelos según el coste del peaje.

Se divide la autopista en dos tramos bien diferenciados, uno norte que va desde el límte de Castellón a Sagunto, y otro sur desde Algemesí hasta El Campello. Además, se tienen en cuenta las actuaciones que pretenden llevarse a cabo en las carreteras afectadas según el plan de inversión estatal PITVI.

Para poder comparar los diferentes escenarios propuestos se plantea la creación de un ratio, definiéndose así el 'ratio específico' que compara económicamente las vías a través de la movilidad de vehículos que se experimenta en cada carretera por unidad de longitud, frente a los costes que conlleva su explotación (peaje), conservación e inversión de las nuevas actuaciones previstas.

Al final se obtienen las conclusiones que ayuden a las administraciones afectadas sobre la decisión a tomar en la renovación o no de la concesión de la AP-7 a partir del año 2020.

\section{INTRODUCCIÓN}

La autopista AP-7 forma parte del corredor viario mediterráneo, uno de los grandes corredores viarios de la Unión Europea. Este corredor comunica Francia con Algeciras, está actualmente en régimen de concesión que se extingue en 2019. 
La repercusión económica que tiene el viario en el sistema productivo de la Comunidad Valenciana es evidente históricamente, ya que ha potenciado muchos sectores productivos de la misma, como por ejemplo los sectores turístico (zonas costeras de toda la Comunidad) e industrial (sector de la cerámica en Castellón). Por ello, se pretende realizar una comparación de la variación del impacto económico en el sistema viario afectado de la Comunidad Valenciana ante una reducción de las tarifas del peaje o su supresión total.

El corredor viario implicado en el estudio se divide en dos tramos diferenciados: norte y sur. En el tramo sur, la función de la AP-7 desde Algemesi hasta El Campello es dar acceso a la costa valenciana, donde el tráfico se apoya también en la actual nacional N-332 y autovía A-38 (figura 1). En este tramo existen también dos alternativas más atractivas por el interior para viajes de largo recorrido que la autopista: uno es la autovía A-35 con la autovía A-7 por Alcoy. La otra es el itinerario formado por la autovía A-35, A-33 y A-31, por la Font de la Figuera. Estos itinerarios son los elegidos por los vehículos que viajan desde Alicante hasta Valencia.

En el tramo Norte se observa preferentemente tráfico de largo recorrido desde Sagunto hasta el límite de provincia con Tarragona. Este tramo está formado por dos posibles itinerarios principales: por un lado, el itinerario de la AP-7; por otro lado, el itinerario de la autovía A-7 con la autovía CV-10 y las carreteras CV-13 y N-340.

Para facilitar el procesamiento de los datos y el seguimiento del estudio, se subdivide cada uno de los dos corredores en cuatro tramos menores. De este modo se analizan mejor los tráficos y la funcionalidad de cada uno. (Ver tabla 1).

\begin{tabular}{|c|c|c|c|c|}
\hline Tramo & SUBTRAMO & Inicio aprox. & FIN aprox. & Infraestructuras consideradas \\
\hline \multirow{4}{*}{ Norte } & I & Vinaroz & Torreblanca & N-340, CV-10, CV-13, AP-7 \\
\cline { 2 - 5 } & II & Torreblanca & Castellón & N-340, CV-10, AP-7 \\
\cline { 2 - 5 } & III & Castellón & Nules & N-340, CV-10, AP-7 \\
\cline { 2 - 5 } & IV & Nules & Sagunto & N-340, A-7, AP-7 \\
\hline \multirow{4}{*}{ Sur } & I & Picassent & Gandía & N-332, A.38, AP-7 \\
\cline { 2 - 5 } & II & Gandía & Denia & N-332, AP-7 \\
\cline { 2 - 5 } & III & Denia & Benidorm & N-332, AP-7 \\
\cline { 2 - 5 } & IV & Benidorm & El Campello & N-332, AP-7 \\
\hline
\end{tabular}

Tabla 1.- Definición de los subtramos de cada uno de los corredores viarios mediterráneos de la Comunidad Valenciana. 


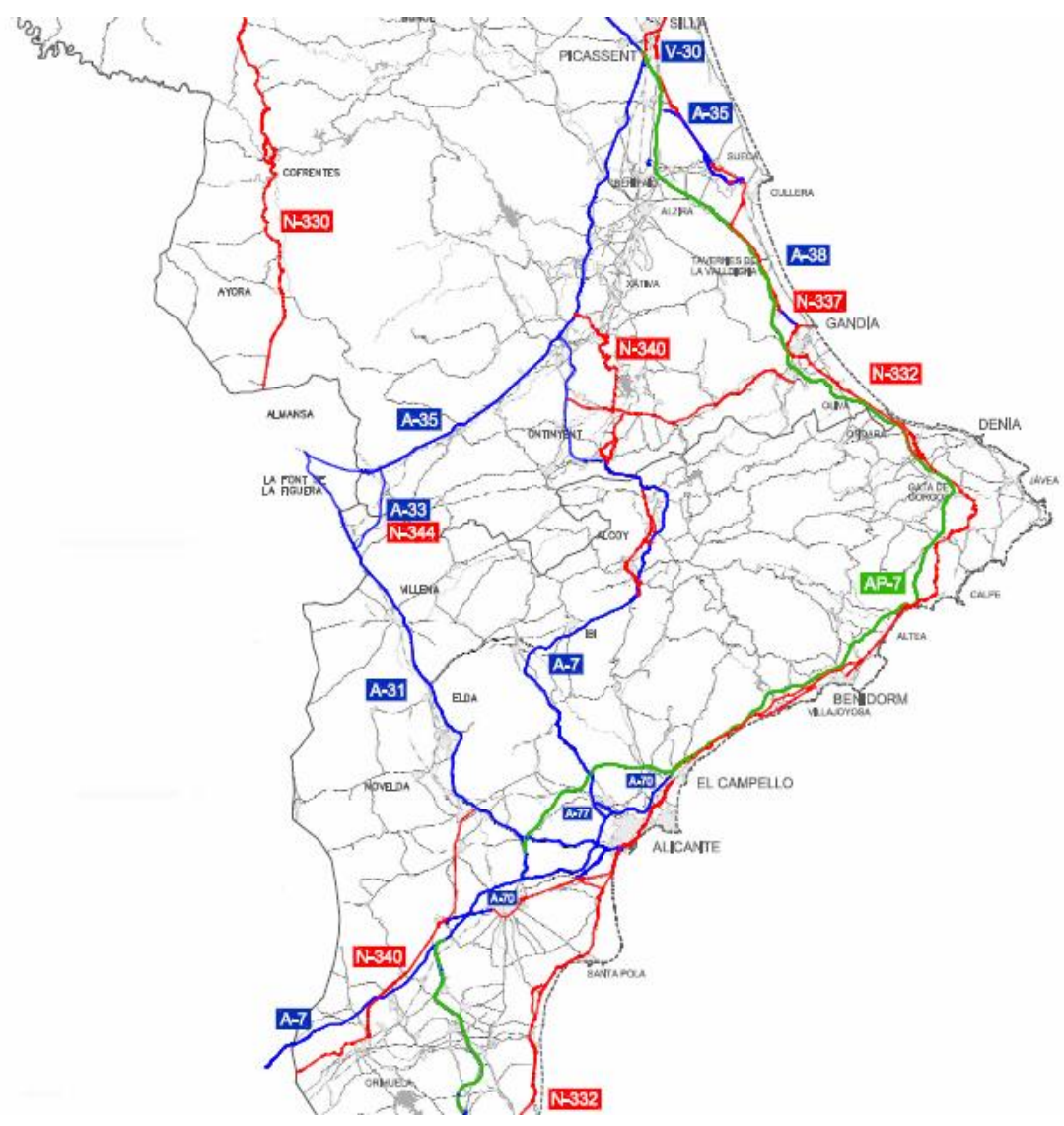

Figura 1. Mapa de carreteras del corredor viario Sur en la Comunidad Valenciana.

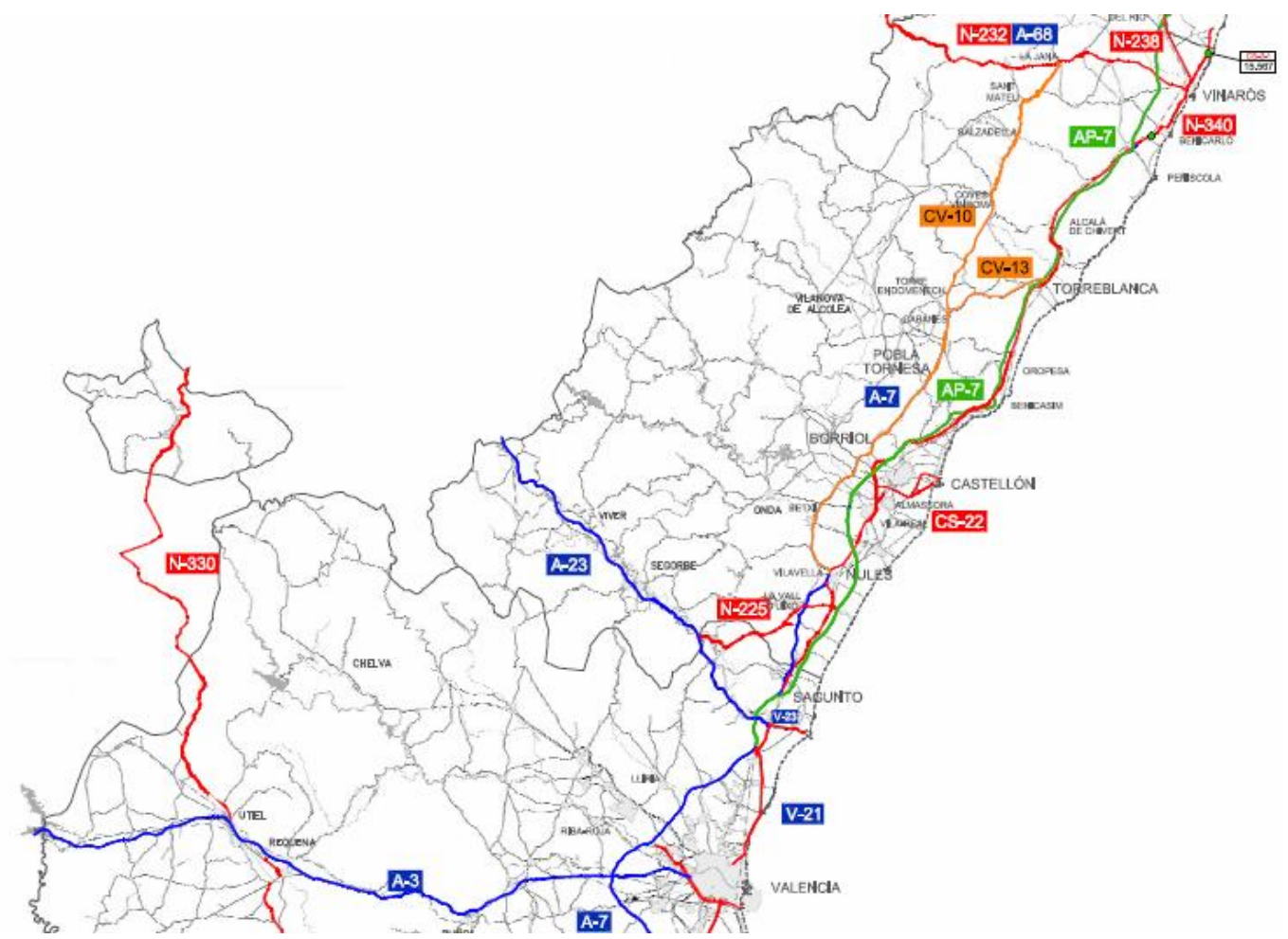

Figura 2. Mapa de carreteras del corredor viario Norte en la Comunidad Valenciana. 


\section{METODOLOGÍA.}

La metodología a seguir será, inicialmente, proponer los escenarios que van a considerarse para cada una de las situaciones presentes y futuras, que se podrán dar en el sistema viario del corredor mediterráneo debido a la renovación o no de la concesión de la AP-7 en el año 2019. A continuación, se analizan los tráficos viarios [I] y [II], en cada escenario y para cada año, definiéndose el año origen el 2013, el año de servicio 2024 y el año horizonte 2040, para analizar el impacto económico de cada escenario.

Se obtienen por un lado la movilidad del corredor en cada uno de los escenarios definidos y se obtienen también los costes de conservación y explotación de los mismos; para posteriormente, a través del ratio específico analizar los resultados.

\section{RESULTADOS.}

\subsection{Definición de los escenarios.}

Se define el escenario 0 'Situación actual' que se proyectará para el año de servicio 2024 donde los peajes de la autopista AP-7 se mantienen según las condiciones tarifarias actuales. Se establecen las siguientes hipótesis:

- No existe trasvase de tráfico hacia la AP-7 desde las diferentes infraestructuras que conforman los corredores.

- No se han ejecutado las actuaciones contempladas en el PITVI 2014 - 2024 (Plan de Infraestructuras, Transporte y Vivienda 2012-2024) para la Comunidad Valenciana). [III]. Con las mismas hipótesis más la realización de actuaciones nuevas de mejora propuestas por el concesionario [IV], se define el escenario 1 'Renovación de la concesión de la AP-7 con las condiciones actuales', para el año horizonte 2040.

Para el escenario 2 'Renovación de la concesión de la AP-7 con peaje blando' se estudian los años 2024 y 2040, y los peajes de la autopista AP-7 reducen su coste un 50\%. Se considera un trasvase de tráfico desde otras vías del orden del $10 \%$ al $20 \%$, se ejecutan las actuaciones del concesionario [IV] y las contempladas en el plan PITVI [V]. De estas hipótesis resultarán cuatro subescenarios:

Escenario 2.a: Año horizonte 2024. Trasvase máximo de tráfico del 10\%.

Escenario 2.b: Año horizonte 2024. Trasvase máximo de tráfico del $20 \%$.

Escenario 2.c: Año horizonte 2040. Trasvase máximo de tráfico del 10\%.

Escenario 2.a: Año horizonte 2040. Trasvase máximo de tráfico del 20\%.

Se define el escenario 3 'Liberación del peaje de la AP-7' de la misma forma que el escenario 2, pero con trasvases de tráfico del $30 \%$ al $40 \%$, generando los siguientes subescenarios: 
Escenario 3.a: Año horizonte 2024. Trasvase máximo de tráfico del 30\%.

Escenario 3.b: Año horizonte 2024. Trasvase máximo de tráfico del 40\%.

Escenario 3.c: Año horizonte 2040. Trasvase máximo de tráfico del 30\%.

Escenario 3.d: Año horizonte 2040. Trasvase máximo de tráfico del $40 \%$.

\subsection{Determinación de las IMD (Intensidades medias diarias) por tramos de cada carretera analizada.}

Para conocer la distribución de tráfico en las diferentes infraestructuras viarias del corredor, en los tramos y subtramos, se recopila la información de los mapas de tráfico y aforo [I] y [II] del año 2013.

Para calcular la intensidad media diaria (IMD) futuras de cada vial se emplea la fórmula (1), en vehículos / día:

$$
\operatorname{IMD}_{\text {año i }}=\operatorname{IMD} 2014 *\left(1+\frac{\% \text { Tasa crecimeinto anual }}{100}\right)^{(\text {Año i-2014 })}
$$

Se toma la tasa de crecimiento que dispone la Orden FO/3317/2010 [VI] a utilizar en los estudios de tráfico reflejados en la siguiente tabla 2.

\begin{tabular}{|c|c|}
\hline \multicolumn{2}{|c|}{ Tasa de crecimiento a utilizar en los estudios de tráfico } \\
\hline Período & Incremento anual acumulativo \\
\hline $2010-2012$ & $1,08 \%$ \\
\hline $2013-2016$ & $1,12 \%$ \\
\hline
\end{tabular}

Tabla 2. - Tasa de crecimiento anual utilizados en los estudios de tráfico.

Por tanto la IMD de 2017 se determina con la tasa de crecimiento anual de 1,12\% y para las de IMD de 2024 y 2040 con la tasa de 1,44\%. Los valores que se obtienen se recogen en el trabajo [VII] para cada escenario estudiado con su trasvase de tráfico correspondiente.

\subsection{Determinación de la movilidad de cada tramo.}

La movilidad (en millones de vehículos anuales y kilómetro) en cada infraestructura se obtiene con la fórmula (2):

$$
\text { Movilidad }=\mathrm{L} \times \mathrm{IMD} \times 365 \times 10^{-6}
$$

Siendo: L= longitud del tramo de infraestructura viaria (kilómetros); IMD (vehículos/día). Los valores obtenidos se recogen en [VII]. 


\subsection{Costes asociados a cada infraestructura.}

Los costes que se asocian a cada infraestructura los conforman:

- Los costes de explotación (peaje) en la autopista AP-7 en concesión. Se consideran en los escenarios 0,1 y 2 .

- Los costes de conservación de las infraestructuras viarias: se consideran todas las carreteras y autovías. En el escenario 3, en la AP-7 se consideran los costes de conservación en lugar de los costes de peaje ya que se supone el retorno de la titularidad al Estado.

- Los costes de inversión de infraestructuras viarias contempladas en el plan PITVI 20122024.

\subsubsection{Costes de explotación (peaje).}

El coste de la autopista AP-7 es el importe del peaje directo que abonan los usuarios por su uso. Este coste se determina a partir de las tarifas existentes en 2015 en cada uno de los tramos, aplicadas a las IMD estimadas en cada uno de ellos. Se diferencia el importe para vehículos ligeros y para pesados, según las tarifas vigentes. Las tarifas se consideran constantes en el tiempo, pero sin el $21 \%$ de IVA, ya que son impuestos que repercuten finamente en el Estado.

El cálculo del coste del peaje se ha realizado tramo a tramo, lo que implica que su importe sea un 5\% superior frente al coste del tramo completo sin realizar entradas y salidas. No se consideras aumentos del coste debido al IPC, ni a cláusulas de revisión de la ampliación de la concesión en su caso, ya que ésta se extingue en el año 2019.

Los valores obtenidos se recogen en [VII].

\subsubsection{Costes de conservación.}

Los costes de conservación en cada uno de los tramos de las infraestructuras viarias se han obtenido considerando:

- "Recomendaciones para la evaluación económica, coste-beneficio de estudios y proyectos de carreteras. Actualización del valor de tiempo y costes en accidentes y combustibles", redactado por el MOPU en octubre de 1990, considerando la actualización de costes.

- La recomendación del Banco Mundial según la cual cada país debe dedicar anualmente entre el 2 y el 3\% del valor patrimonial de sus carreteras a su conservación. En el año 2000, España dedicaba entorno al 1,1-1,2\% a conservar sus infraestructuras viarias. Aunque el Plan COEX del Ministerio de Fomento pretendía subir el gasto en conservación al 2,2\% en años posteriores, la realidad es que la crisis que se lleva sufriendo desde 2007 ha provocado la obtención de valores inferiores a lo esperado. Esto puede hacerse extensible a las comunidades 
autónomas en particular a la valenciana.

Por todo lo expuesto los costes de conservación de las infraestructuras viarias son:

- Carreteras convencionales de un carril por sentido: $\quad 20000 €$ anuales / Km

- Autopistas y autovías de dos carriles por sentido: $\quad 45000 €$ anuales $/ \mathrm{Km}$

Es necesario especificar que en el escenario 3, se consideran los costes de conservación de la autopista AP-7, ya que en este supuesto la concesión revierte al Estado.

Los valores obtenidos se recogen en [VII].

\subsubsection{Costes de inversión.}

Para calcular los costes de inversión en cada tramo y subtramo se consideran únicamente las infraestructuras viarias que intervienen en el corredor Mediterráneo y que se recogen en el Plan PITVI 2012-2024.

El Plan de Infraestructuras, Transporte y Vivienda, impulsado por el Ministerio de Fomento, recoge una serie de inversiones para mejorar el sistema de transporte y su competitividad a nivel estatal. En él, se recogen las partidas dedicadas a cada modo de transporte y en sus infraestructuras (ferrocarril, carretera, avión, barco...).

Se han cuantificado y presupuestado todas las actuaciones que el Plan pretende llevar a cabo dentro del corredor Mediterráneo a su paso por la Comunidad Valenciana. El total de las inversiones en las infraestructuras viarias consideradas es de 1 572,17 millones de euros. Esta suma se reparte en un período de 28 años comprendido entre 2012 (inicio del Plan) y 2040 (año horizonte).

Se ha repercutido cada inversión dentro del tramo y subtramo para cada escenario. Así, en los 28 años considerados, la inversión anual de las actuaciones del Plan asciende a 56,15 millones de euros: 17,95 millones de $€$ en el corredor norte y 38,20 millones de $€$ en el corredor sur.

De este modo, al sumar todos los costes obtenidos para cada infraestructura viaria se obtienen los costes totales para cada subtramo, tramo y, finalmente, escenario. Los valores obtenidos se recogen en la tabla 3 a continuación: 


\begin{tabular}{|c|c|c|c|c|c|c|c|}
\hline \multicolumn{8}{|c|}{ Suma de costes de explotación (peaje), conservación e inversión (millones de euros, M€) } \\
\hline & Tramo & S I & S II & S III & S IV & Total tramo & Total escenario \\
\hline \multirow{2}{*}{ E0 } & Norte & 18,49 & 26,79 & 28,72 & 20,64 & 94,64 & \multirow[b]{2}{*}{202,09} \\
\hline & Sur & 31,51 & 10,35 & 25,90 & 27,17 & 94,93 & \\
\hline \multirow{2}{*}{ E1 } & Norte & 28,54 & 41,01 & 38,88 & 29,86 & 138,6 & \multirow[b]{2}{*}{300,32} \\
\hline & Sur & 50,60 & 22,55 & 49,02 & 39,60 & 161,77 & \\
\hline \multirow{2}{*}{ E2.a } & Norte & 17,78 & 26,99 & 24,71 & 18,83 & 88,31 & \multirow[b]{2}{*}{188,25} \\
\hline & Sur & 31,77 & 15,85 & 31,234 & 21,09 & 99,95 & \\
\hline \multirow{2}{*}{ E2.b } & Norte & 18,76 & 30,08 & 29,83 & 18,83 & 97,49 & \multirow[b]{2}{*}{203,90} \\
\hline & Sur & 35,29 & 16,37 & 32,25 & 22,50 & 106,41 & \\
\hline \multirow{2}{*}{ E2.c } & Norte & 20,34 & 31,29 & 29,92 & 22,19 & 103,74 & \multirow[b]{2}{*}{217,36} \\
\hline & Sur & 36,76 & 17,25 & 34,726 & 24,89 & 113,62 & \\
\hline \multirow{2}{*}{ E2.d } & Norte & 21,56 & 35,17 & 36,36 & 25,39 & 118,49 & \multirow{2}{*}{240,23} \\
\hline & Sur & 41,17 & 17,90 & 36,00 & 26,66 & 121,74 & \\
\hline \multirow{2}{*}{ E3 } & Norte & 9,56 & 12,02 & 5,19 & 6,72 & 33,49 & \multirow{2}{*}{86,96} \\
\hline & Sur & 14,41 & 11,86 & 19,62 & 7,58 & 53,47 & \\
\hline
\end{tabular}

Tabla 3. - Costes totales de cada escenario, explotación más peaje más conservación en millones de euros, M€.

\section{ANALISIS DE RESULTADOS.}

\section{1. Ratio económico.}

Para analizar los resultados obtenidos se define el "ratio específico" (miles de euros / (vehículos por kilómetro) como la suma de los costes totales asociados a cada vía divido entre su movilidad en los distintos escenarios según la fórmula 3. Este ratio refleja los miles de euros que le cuesta a la sociedad cada vehículo por kilómetro en las carreteras estudiadas.

$$
\text { Ratio específico }=\frac{\text { Suma de costes peaje,conservación e inversión }}{(\text { Movilidad } \times 1000)}
$$

Donde los costes están en millones de euros y la movilidad en millones de vehículos anuales por kilómetro.

Por tanto, para cada escenario se deduce que si el parámetro muestra valores menores que en el origen (escenario 0 para los años horizontes 2024 y escenario 1 para los años horizontes 2040), el supuesto sería beneficioso para la sociedad desde el punto de vista económico. Por el contrario, si el valor del parámetro aumenta, significa que la sociedad tiene que invertir más miles de euros por vehículo y kilómetro al realizar las actuaciones y cumplir las hipótesis planteadas para cada escenario.

En la tabla 4 se muestra un resumen de los resultados del ratio específico en cada escenario. 


\begin{tabular}{|c|c|c|c|c|c|c|c|}
\hline \multicolumn{8}{|c|}{ Ratio específico (Miles de euros / vehículo y kilómetro) } \\
\hline & Tramo & S I & S II & S III & S IV & Total tramo & Total escenario \\
\hline \multirow{2}{*}{ E0 } & Norte & 34,54 & 35,29 & 50,56 & 42,88 & 40,38 & \multirow[b]{2}{*}{41,91} \\
\hline & Sur & 48,72 & 23,14 & 48,80 & 49,00 & 43,56 & \\
\hline \multirow{2}{*}{ E1 } & Norte & 34,76 & 34,14 & 48,43 & 49,53 & 41,08 & \multirow[b]{2}{*}{46,80} \\
\hline & Sur & 60,17 & 33,20 & 59,38 & 56,82 & 53,17 & \\
\hline \multirow{2}{*}{ E2.a } & Norte & 37,72 & 32,37 & 43,95 & 37,17 & 37,17 & \multirow[b]{2}{*}{39,13} \\
\hline & Sur & 44,45 & 29,92 & 48,72 & 38,34 & 41,04 & \\
\hline \multirow{2}{*}{ E2.b } & Norte & 34,34 & 38,31 & 53,54 & 37,17 & 40,70 & \multirow[b]{2}{*}{42,11} \\
\hline & Sur & 46,70 & 31,49 & 51,49 & 41,22 & 43,48 & \\
\hline \multirow{2}{*}{ E2.c } & Norte & 25,14 & 27,40 & 38,05 & 35,12 & 30,72 & \multirow[b]{2}{*}{33,67} \\
\hline & Sur & 40,90 & 25,91 & 42,18 & 36,00 & 36,90 & \\
\hline \multirow{2}{*}{ E2.d } & Norte & 26,70 & 30,51 & 47,04 & 40,41 & 35,16 & \multirow[b]{2}{*}{37,16} \\
\hline & Sur & 43,35 & 27,40 & 44,66 & 38,86 & 39,33 & \\
\hline \multirow{2}{*}{ E3.a } & Norte & 17,47 & 15,48 & 9,39 & 14,19 & 14,29 & \multirow[b]{2}{*}{18,01} \\
\hline & Sur & 17,95 & 23,25 & 32,03 & 13,98 & 21,66 & \\
\hline \multirow{2}{*}{ E3.b } & Norte & 17,28 & 15,15 & 9,47 & 14,19 & 14,18 & \multirow[b]{2}{*}{17,89} \\
\hline & Sur & 16,89 & 23,71 & 33,03 & 14,09 & 21,52 & \\
\hline \multirow{2}{*}{ E3.c } & Norte & 12,00 & 10,80 & 6,83 & 11,29 & 10,28 & \multirow[b]{2}{*}{13,58} \\
\hline & Sur & 14,21 & 18,50 & 24,94 & 11,12 & 17,10 & \\
\hline \multirow{2}{*}{ E3.d } & Norte & 12,03 & 10,79 & 6,96 & 9,58 & 10,02 & \multirow[b]{2}{*}{13,41} \\
\hline & Sur & 13,44 & 18,86 & 25,46 & 11,21 & 17,00 & \\
\hline
\end{tabular}

Tabla 4.- Ratio específico para cada escenario en millones de euros por vehículo y kilómetro.

Es posible que estos valores así expresados no nos digan nada, pero si reordenamos los valores obtenidos según años horizontes tal y como se muestra en las figuras 1 y 2, podrán observarse variaciones significativas:

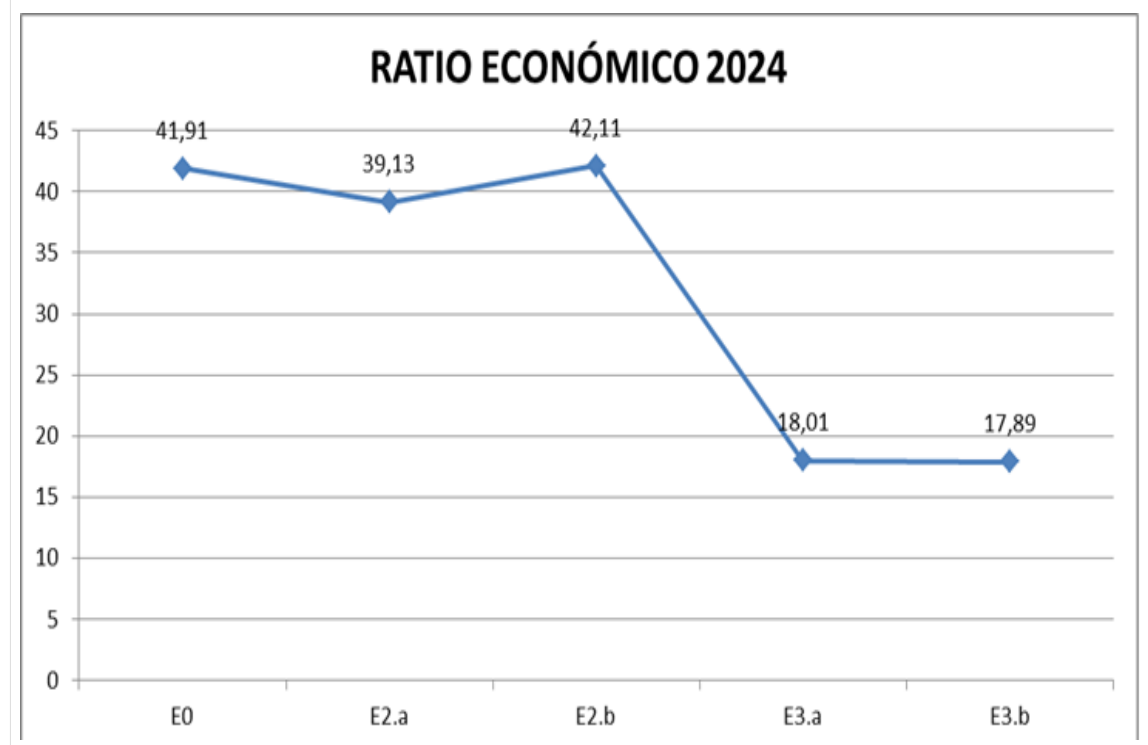

Figura 3. Ratio específico para los escenarios del año 2024 en millones de euros por vehículo y kilómetro. 


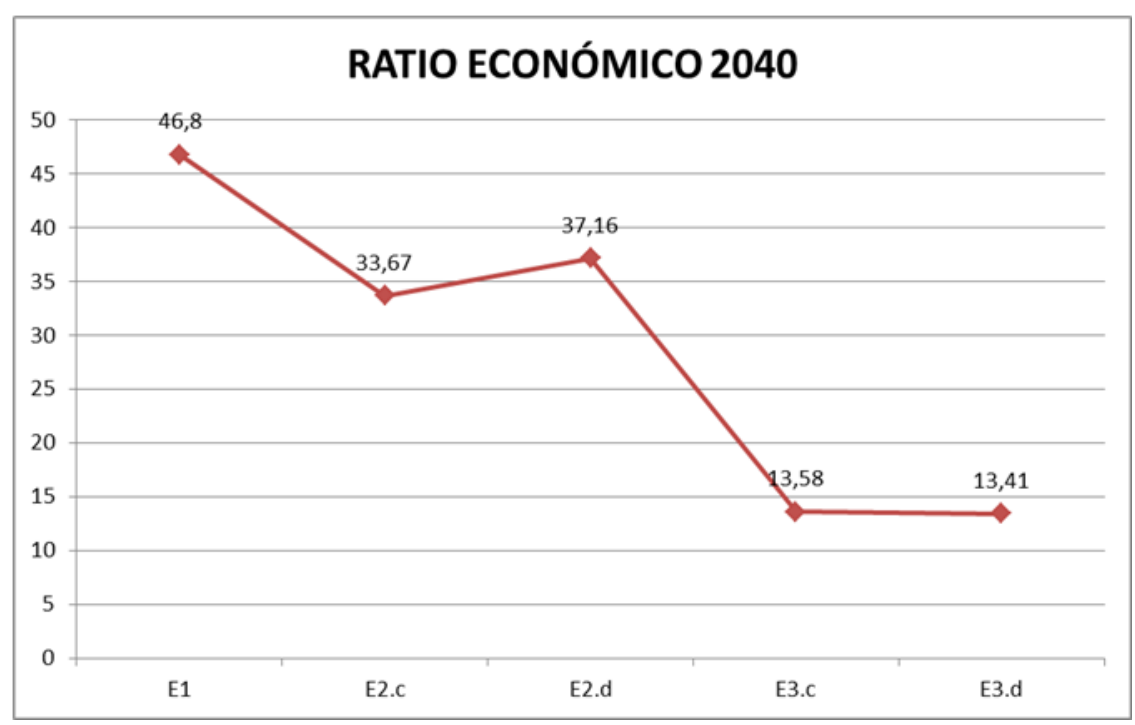

Figura 4. Ratio específico para los escenarios del año 2040 en millones de euros por vehículo y kilómetro.

\section{CONCLUSIONES.}

Se aprecia que los mayores costes totales corresponden al escenario 1 'Renovación de la concesión de la AP-7 con las condiciones actuales' y los menores al escenario 3 'Liberación del peaje de la AP-7'. Esta diferencia se debe a la alta influencia del coste del peaje respecto a los costes totales.

El coste total de la autopista AP-7 por tarifas es de 225,30 millones de euros en el escenario 1 y por conservación del escenario 3 es de 30, 81 millones de euros.

El mejor escenario según el ratio económico es el 3.d que consiste en liberalización de la AP-7, con un valor de 13,41 m€/vh-km. El peor escenario es el 1 con un valor de 46,8 $\mathrm{m} € / \mathrm{vh}-\mathrm{km}$. Por ello se aconseja escoger la liberación del peaje (escenario 3.d).

\section{AGRADECIMIENTOS}

Por su colaboración en la obtención de datos al ingeniero de caminos, canales y puertos D. Manuel Cánovas Carreño por su ayuda inestimable. 


\section{REFERENCIAS BIBLIOGRÁFICAS}

[I] MINISTERIO DE FOMENTO (2014). Plan de Infraestructuras, Transporte y Vivienda 2012-2024 (PITVI), Madrid.

[II] ABERTIS AUTOPISTAS (2013). Documento "Mejora del corredor TarragonaValencia-Alicante. Propuesta de actuaciones".

[III] CONSELLERIA DE INFRAESTUCTURAS, TERRITORIO Y MEDIO AMBIENTE (2014). “Memoria Anual d'Aforamens 2013”, Valencia.

[IV] DIRECCIÓN GENERAL DE CARRETERAS DEL MINISTERIO DE FOMENTO (2014). “Mapas de tráfico de 2013”, Madrid.

[V] PÉREZ ZAMORA, J.E. (2015). "Estudio técnico - económico de la variación de la concesión de la AP-7 en el corredor viario del Mediterráneo en la Comunidad Valenciana.". Trabajo de Final de Grado. Escuela Técnica Superior de Ingenieros de Caminos, Canales y Puertos. Universidad Politécnica de Valencia, Valencia.

[VI] DIRECCIÓN GENERAL DE CARRETERAS DEL MINISTERIO DE FOMENTO (2010). “Instrucción sobre las medidas específicas para la mejora de la eficiencia en la ejecución de las obras públicas de infraestructuras ferroviarias, carreteras y aeropuertos". Orden FOM/3317/2010, Madrid.

[VII] ABERTIS AUTOPISTAS (2015). "Peajes 2015 de la autopista AP-7 (La Jonquera - Alicante)".

[VIII] SERVICIO DE PLANEAMIENTO DE LA DIRECCIÓN GENERAL DE CARRETERAS DEL MINISTERIO DE FOMENTO (1993). "Recomendaciones para la evaluación económica, coste beneficio de estudios y proyectos de carreteras", Madrid.

[IX] TRANSPORTATION RESEARCH BOARD (2010). Highway Capacity Manual 2000. National Research Council, Washington D.C.

[X] DIRECCIÓN GENERAL DE CARRETERAS DEL MINISTERIO DE FOMENTO (2000). “Instrucción de Carreteras: Norma 3.1-IC. Trazado”, Madrid. 